\title{
The clinical value of regular thyroid function tests during amiodarone treatment
}

\section{Stan Benjamens', Robin P F Dullaart', Wim J Sluiter', Michiel Rienstra², Isabelle $\mathbf{C}$ van Gelder $^{2}$ and Thera P Links ${ }^{1}$}

${ }^{1}$ Department of Internal Medicine, Division of Endocrinology and 2Department of Cardiology, University of Groningen, University Medical Center Groningen, Groningen, The Netherlands

\author{
Correspondence \\ should be addressed \\ to $T$ P Links \\ Email \\ t.p.links@umcg.nl
}

\begin{abstract}
Objective: Amiodarone is used for the maintenance of sinus rhythm in patients with arrhythmias, but thyroid dysfunction (amiodarone-induced thyrotoxicosis (AIT) or amiodarone-induced hypothyroidism (AIH)) is a common adverse effect. As the onset of AIT and AIH may be unpredictable, the value of long-term regular monitoring of amiodarone treated patients for thyroid dysfunction is still uncertain.

Design: We retrospectively documented the frequency at which overt thyroid dysfunction was preceded by subclinical thyroid dysfunction.

Methods: We included 303 patients treated with amiodarone between 1984 and 2007. AIT was defined as a lowered TSH level with an elevated free thyroxine (FT4) and AIH was defined as an elevated TSH level with a decreased or subnormal FT4. Subclinical AIT was defined as a lowered TSH level with a normal FT4 and subclinical AIH as an elevated TSH level with a normal FT4.

Results: 200 men and 103 women, aged $62 \pm 12.0$ years, suffering from atrial (260) or ventricular (43) arrhythmias, were evaluated. During a median follow-up of 2.8 (1.0-25) years, 44 patients developed AIT and 33 AlH. In 42 (55\%) patients who developed AIT/AIH, earlier thyroid function tests showed no subclinical AIT or subclinical AIH. In 35 (45\%) patients, AIT/AIH was preceded by subclinical AIT or subclinical AIH (16/44 for AIT and 19/33 for AIH).

Conclusions: In a considerable proportion of patients who developed AIT/AIH, earlier thyroid function tests showed no subclinical AIT/AIH. Less than half of the patients with a subclinical event subsequently developed overt AIT/AIH. This study provides data to reconsider the yield of regular testing of thyroid function to predict overt thyroid dysfunction in amiodarone treated patients.
\end{abstract}

\section{Introduction}

Amiodarone has been shown to be superior to other drugs in maintaining sinus rhythm in patients with symptomatic atrial fibrillation (AF) and to prevent recurrent ventricular arrhythmias $(1,2,3,4)$. However, it is well known that amiodarone is frequently associated with thyroid dysfunction.

The large amount of iodine in amiodarone enhances the iodine exposure to the thyroid, and results in increased iodine content of the thyroid gland. The thyroid autoregulation does not in all patients prevent the effect of an
() 2017 European Society of Endocrinology Printed in Great Britain
European Journal of

Endocrinology

(2017) 177, 9-14 increased iodine exposure to the thyroid, which causes amiodarone-induced thyrotoxicosis (AIT) or amiodaroneinduced hypothyroidism (AIH). AIT is categorized as type 1 when underlying thyroid abnormalities are present (autonomous multinodular goiter or latent Graves' disease), and type 2 when a destructive thyroiditis is caused by the cytotoxic effects of amiodarone (5). A higher occurrence of AIT type 2 (79\%), compared to AIT type 1 (21\%), has been described in a cohort of 200 patients (5). Both subtypes are important clinical issues, 
as they may cause several clinical symptoms or major adverse cardiovascular events, and sometimes be therapy resistant (6). On the other hand, AIH is considered to be of limited clinical risk and can be managed easily by thyroid hormone replacement (7). In most European countries, where iodine intake is low, the proportion of AIT (75\%) is higher compared to AIH (25\%), whereas in North America, where iodine intake is high, the proportion of AIH (66\%) is higher compared to that of AIT (34\%) (8). In the Netherlands, previous studies show an incidence between 8 and 12.2\% for AIT and an incidence between 6 and $6.9 \%$ for $\operatorname{AIH}(9,10,11,12)$.

The value of regularly monitoring amiodarone treated patients for thyroid dysfunction is unclear, mainly because the onset of dysfunction may be unpredictable $(10,11)$. The guidelines of the American Thyroid Association (ATA) and the American Association of Clinical Endocrinologists (AACE) recommend performing thyroid function tests before, at 1 and 3 months after the initiation of amiodarone therapy, and at 3-6 month intervals thereafter $(13,14)$. In contrast, the guidelines of The Dutch Society of Internal Medicine do not recommend thyroid function tests during amiodarone therapy (15).

The present study was initiated to describe the yield of regular thyroid function tests during amiodarone treatment by determining the number of cases in which overt thyroid dysfunction was preceded by a subclinical thyroid event.

\section{Patients and methods}

We studied 303 predominantly white patients treated with amiodarone at the University Medical Center of Groningen, The Netherlands between 1984 and 2007. Ahmed et al. included the majority of these patients (265) in a retrospective evaluation of patients on their first period of amiodarone treatment for documented cardiac arrhythmias; thyroid function tests were performed at baseline prior to amiodarone initiation and then every 6 months (10). The remaining 38 patients participated in the prospective CONVERT study, including patients in the presence of symptomatic AF recurrence; thyroid function tests were performed at the outpatient department upon inclusion, then after 2 weeks of amiodarone loading, and then again every 4 months until 2 years following randomization (9).

In both studies only patients with normal thyroid function prior to amiodarone initiation, as established by thyroid function tests, were included. After ending both
Table 1 Biochemical definitions of (sub)clinical AIT and AIH.

\begin{tabular}{|c|c|c|c|c|}
\hline & & 1984-1999* & 1999-2006 * * & $2006-2014$ * * * \\
\hline \multirow[t]{2}{*}{ AIT } & $\mathrm{TSH}$ & $\leq 0.13$ & $\leq 0.22$ & $\leq 0.25$ \\
\hline & FT4 & $\geq 26.0$ & $\geq 18.2$ & $\geq 19.5$ \\
\hline \multirow[t]{2}{*}{ Subclinical AIT } & TSH & $0.13-0.30$ & $0.22-0.45$ & $0.25-0.50$ \\
\hline & FT4 & $9.0-26.0$ & $6.3-18.2$ & $11.0-19.5$ \\
\hline \multirow[t]{2}{*}{ AlH } & $\mathrm{TSH}$ & $\geq 6.5$ & $\geq 8.7$ & $\geq 10.0$ \\
\hline & FT4 & $\leq 9.0$ & $\leq 6.3$ & $\leq 11.0$ \\
\hline \multirow[t]{2}{*}{ Subclinical AlH } & $\mathrm{TSH}$ & $3.00-6.50$ & $3.50-8.70$ & $4.00-10.00$ \\
\hline & FT4 & $9.0-26.0$ & $6.3-18.2$ & $11.0-19.5$ \\
\hline
\end{tabular}

${ }^{*}$ Amersham Amerlite ${ }^{\oplus}$, in IU/L; ** Delfia $^{\odot}$, in $\mathrm{mU} / \mathrm{L}^{*}{ }^{* * *}$ Roche Diagnostics $^{\odot}$, in $\mathrm{mU} / \mathrm{L}$.

studies, patients were followed in the outpatient clinic of the Department of Cardiology and, according to the local protocol, thyroid function tests were performed every six months, and more frequently when subclinical results were diagnosed. We collected patients' sex; date of birth; time and cause of death; start and end of amiodarone therapy (or continuation after end of follow-up); all available TSH and FT4 results; possible therapy for AIT/AIH; and documented side-effects of amiodarone treatment, by screening patients' charts retrospectively (from 2007 until 2014) at the Department of Cardiology. Laboratory values for FT3 were not included due to a high frequency of missing values.

The primary outcome in this study was the occurrence of overt and/or subclinical thyroid dysfunction. We have defined AIT, in accordance with the definitions of Ahmed et al. as a suppressed TSH in combination with an elevated FT4 (10). AIH has been defined as a TSH above the institutional range in combination with a decreased or subnormal FT4. We defined subclinical AIT as a TSH below the reference range in combination with a normal FT4 and subclinical AIH as a TSH above the reference range in combination with a normal FT4 (Table 1).

\section{Statistical analysis}

Baseline descriptive statistics, demographics and clinical characteristics are presented as mean \pm SD or median (range) for continuous variables and counts with percentages for categorical variables. Differences between variables were evaluated for continuous data by student's T-test or Mann-Whitney U-test, depending on distribution toward normality. All tests of significance are two-tailed, with $P$-values of $<0.05$ assumed to indicate significance. All statistical analyses were performed using the Statistical Package for the Social Sciences (IBM SPSS Statistics Version 22). 


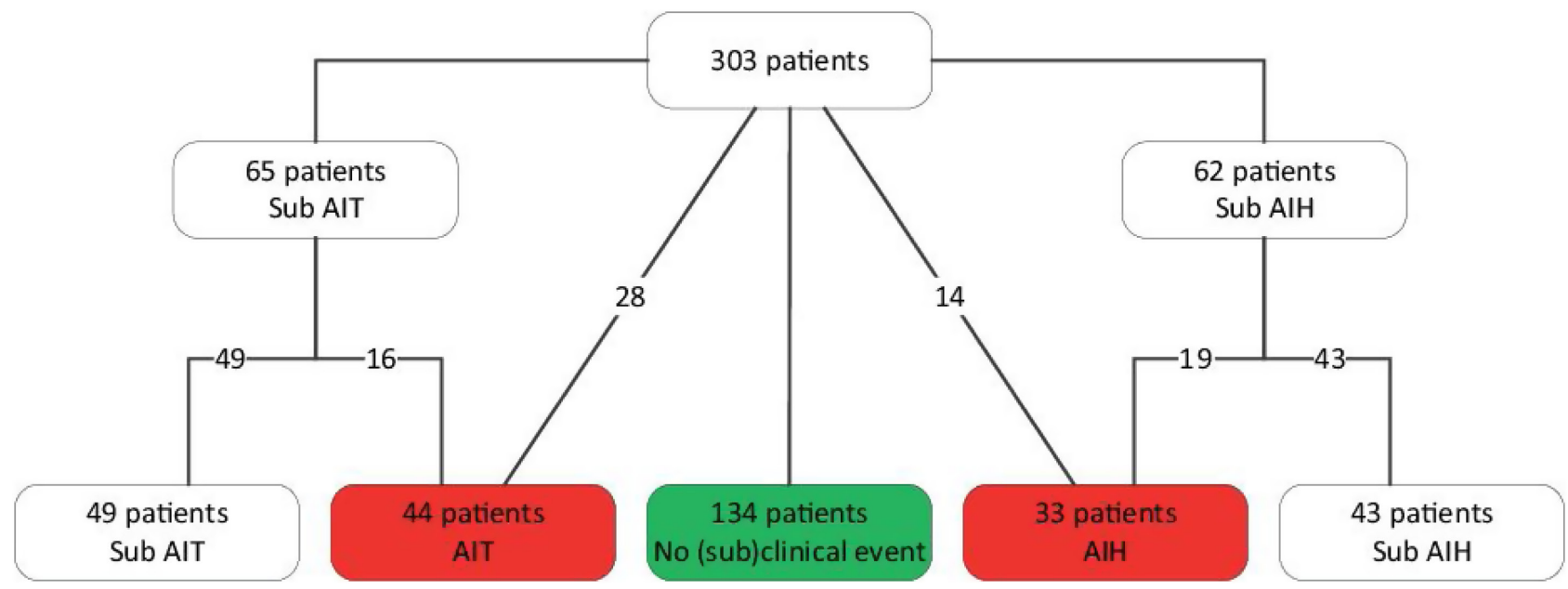

\section{Figure 1}

Patients with (sub)clinical thyroid events and patients without amiodarone-associated thyroid events, focusing on the development of amiodarone-induced thyrotoxicosis (AIT) and amiodarone-induced hypothyroidism (AIH).

\section{Results}

\section{Patient characteristics and outcomes}

We evaluated 303 patients, 200 (66\%) men and 103 (44\%) women, with a mean age of $62.3 \pm 12.0$ years. The indication for amiodarone treatment was AF in 260 (86\%) patients and ventricular arrhythmias in 43 (14\%) patients. The daily maintenance dose of oral amiodarone was $200 \mathrm{mg}$, which was increased or decreased based on serum levels of amiodarone and physicians' decision. The median follow-up was 2.8 (1.0-25) years with an amiodarone use of 1.9 (1.0-24) years; 119 (39\%) patients died during follow-up. 77 out of 303 patients (25\%) developed overt thyroid dysfunction: 47 (61\%) men and 30 (39\%) women. A total of 44 out of $77(57 \%)$ patients developed AIT, and 33 out of 77 (43\%) patients developed AIH.

\section{(Sub)Clinical events}

In 16 out of 44 (64\%) patients who developed AIT this was preceded by subclinical AIT (Fig. 1). In 65 patients who developed subclinical AIT eventually 49 (75\%) developed no AIT within a follow-up period of $3.4(0-16)$ years. Out of 49 patients with subclinical AIT, 27 (55\%) remained subclinical during the two years following the first event and 22 (45\%) spontaneously reverted to euthyroidism in this period. In 19 out of 33 (58\%) patients who developed $\mathrm{AIH}$ this was preceded by subclinical $\mathrm{AIH}$ (Fig. 1). Of the 62 patients who developed subclinical AIH eventually 43 (70\%) developed no AIH within a median follow-up of $2.2(0-13)$ years. The median time between the start of amiodarone and the occurrence of subclinical AIT was 1.4 (0.1-9) years, compared with 2.6 (0.1-9) years for overt AIT. Subclinical AIH occurred after 1.1 (0.1-18) years, compared with $1.7(0.1-24)$ years for overt AIH (Table 2).

\section{Clinical signs}

Twenty-one (48\%) out of 44 patients who developed AIT showed one or multiple AIT-associated clinical signs. Increased sweating was reported in 8 patients, palpitations in 7 patients, weight loss in 6 patients and agitation in 6 patients. AIH patients reported general symptoms of hypothyroidism.

\section{Treatment consequences}

Treatment was started at a median of 46 (1-182) and 84 (13-371) days after the diagnoses of overt AIT in 18 patients and $\mathrm{AIH}$ in 22 patients respectively. Four (10\%) patients with AIT received prednisolone, 6 (14\%) received

Table 2 Outcomes after follow-up.

\begin{tabular}{|c|c|c|}
\hline & Patients, $n(\% *)$ & Time till event, years* * \\
\hline AIT & $44(15)$ & $2.6(0.2-9)$ \\
\hline Subclinical AIT & $65(21)$ & $1.4(0.1-9)$ \\
\hline AlH & $33(11)$ & $1.7(0.1-24)$ \\
\hline Subclinical AIH & $62(20)$ & $1.1(0.1-18)$ \\
\hline
\end{tabular}

*Percentage of total number of included patients, 303; **Values are shown as median (range). 
methimazole and $8(18 \%)$ received a combination of prednisolone and methimazole; 26 (59\%) patients were not treated, and in 10 of them amiodarone was discontinued. The FT4 levels in the non-treated group were significantly lower compared to the treatment group $(P=0.001)$, while TSH levels did not differ $(P=0.084)$. AIH was treated with levothyroxine in $22(67 \%)$ cases and in $11(33 \%)$ cases clinicians decided not to give any medication. Both FT4 and TSH levels did not significantly differ between the treatment and non-treatment groups ( $P=0.059$ and $P=0.637$ respectively).

\section{Discussion}

In this single university center study we evaluated changes thyroid function status in a large group of patients treated with amiodarone, and observed 44 cases of AIT and 33 cases of AIH. For the majority of patients who developed AIT, earlier thyroid function tests showed no signs of subclinical AIT, and in only a small percentage of cases, for AIH the clinical event was preceded by a subclinical event. For both AIT/AIH only a minority of the subclinical events was followed by a clinical event. Our data adds to the discussion of the currently expert-based recommendation that thyroid function should be tested before and at 1 and 3 months following initiation of amiodarone therapy, and at 3-6 month intervals thereafter (12). Ahmed et al. identified the following predictors for amiodaroneassociated thyroid dysfunction: age $>62$ years for AIT and for AIH a baseline TSH $>1.4 \mathrm{mU} / \mathrm{L}$; left ventricular function $<45 \%$; and diabetes mellitus (10). However, it was not possible to identify which patients are prone to develop amiodarone-associated thyroid dysfunction or could be selected for regular thyroid function testing.

The clinical course of AIT/AIH does not support the need for regular surveillance of thyroid function, since adverse outcomes after sudden onset of thyroid dysfunction were neither present in our study population nor found in the literature (16). However, the sudden onset of AIT/AIH could explain our observation that the majority of patients showed an overt event without an earlier subclinical event.

Neither the American guidelines of ATA/AACE nor the guidelines of The Dutch Society of Internal Medicine recommend when and whom to treat in case of AIT/AIH $(13,15)$. In the present retrospective study we showed no differences in TSH and FT4 levels in treated and nontreated AIH patients and neither a significant difference in FT4 levels between the treated and non-treated AIT patients.

The clinical course of AIH is unlikely to require amiodarone withdrawal with no major complications being described with its continued use (7). With regard to AIT, in a randomized clinical trial all AIT type 2 patients reached euthyroidism despite continuation of amiodarone treatment (12). Additionally, three cases of AIT recurrence after continuation of amiodarone showed a relatively mild and spontaneously resolving course (17). Nonetheless, discontinuation can be deemed necessary due to the severity of clinical (cardiovascular) symptoms, as limited data suggest that continuation of amiodarone may be associated with a delayed restoration of euthyroidism $(7,18)$. A recent study showed that all patients who suffered amiodarone-associated side-effects of the thyroid (16.6\%) fully recovered after amiodarone discontinuation or dose reduction, supporting the mild course of thyroid dysfunction in most patients (19). Furthermore, total thyroidectomy was never necessary in our study, in keeping with a previous report. One study showed that thyroid surgery is a valid therapeutic option in a small subgroup (7\% out of 394 patients) of AIT patients with severe left ventricular dysfunction (20). In another study, 8 out of 84 patients underwent a surgical intervention (21). In general, subtotal or total thyroidectomy should be considered only in patients with progressive AIT when patients are resistant to a high-dose prednisolone and watchful medical management could have negative effects $(22,23)$.

In this study, $15 \%$ of patients developed AIT and $11 \%$ of patients developed $\mathrm{AIH}$, a percentage which is slightly higher than that in the data of Trip et al. (AIT $12 \%$ and AIH 6.9\%), but lower than that in the data of Hofmann et al. (AIT $20.8 \%$ and AIH 25.0\%) $(11,24)$. This could be explained by the longer follow-up period in our study compared with that in the study of Trip et al. since the iodine intake has been regarded as comparable; it could also be explained by a high incidence of thyroid dysfunction prior to amiodarone initiation in the study of Hofmann et al. The time between initiation of amiodarone and the occurrence of AIT and AIH in our study is comparable for AIT, but differs for AIH with the study by Trip et al. (11).

The retrospective nature of the present study is its most important limitation, which is the main reason for the lack of FT3 and TPO-Ab laboratory values and the lack of information about the cumulative dose of amiodarone. However, earlier studies show conflicting results with regard to the effect of the daily and/or cumulative dose 
of amiodarone on the occurrence and severity of AIT/ AIH. In addition, in this study no differentiation has been made between AIT type 1 and type 2 . We considered that the majority of patients with AIT would have been type 2 , since their thyroid function was normal at the start of amiodarone treatment. The strength of this study can be found in the high number of patients included and the long period of follow-up.

In conclusion, this study provides data to reconsider the yield of regular testing of thyroid function to predict overt thyroid dysfunction in patients on amiodarone treatment.

\section{Declaration of interest}

The authors declare that there is no conflict of interest that could be perceived as prejudicing the impartiality of this study.

\section{Funding}

This work did not receive any specific grant from any funding agency in the public, commercial, or not-for-profit sector.

\section{Author contribution Statement}

$S$ Benjamens: Acquired the data and was involved in data analysis and interpretation and writing the manuscript. R P F Dullaart: Was involved in data analysis and interpretation, writing the manuscript and contributed to the final adjustments to the manuscript after revising it critically for intellectual content. W J Sluiter: Was involved in data analysis and interpretation, writing the manuscript and contributed to the final adjustments to the manuscript after revising it critically for intellectual content. M Rienstra: Was involved in data analysis and interpretation, writing the manuscript and contributed to the final adjustments to the manuscript after revising it critically for intellectual content. I C van Gelder: Was involved in data analysis and interpretation, writing the manuscript and contributed to the final adjustments to the manuscript after revising it critically for intellectual content. T P Links: Initiated the study and was involved in data analysis and interpretation, writing the manuscript and contributed to the final adjustments to the manuscript after revising it critically for intellectual content.

\section{References}

1 Singh BN, Singh SN, Reda DJ, Tang XC, Lopez B, Harris CL, Fletcher RD, Sharma SC, Atwood JE, Jacobson AK et al. Amiodarone versus sotalol for atrial fibrillation. New England Journal of Medicine 2005352 1861-1872. (doi:10.1056/NEJMoa041705)

2 Zimetbaum P. Amiodarone for atrial fibrillation. New England Journal of Medicine 2007356 935-941. (doi:10.1056/NEJMct065916)

3 Epstein AE, Olshansky B, Naccarelli GV, Kennedy JI Jr, Murphy EJ \& Goldschlager N. Practical Management Guide for Clinicians Who Treat Patients with Amiodarone. American Journal of Medicine 2016 129 468-475. (doi:10.1016/j.amjmed.2015.08.039)

4 Schleifer JW, Sorajja D \& Shen WK. Advances in the pharmacologic treatment of ventricular arrhythmias. Expert opinion on Pharmacotherapy 201516 2637-2651. (doi:10.1517/14656566.2015.1100170)

5 Tomisti L, Rossi G, Bartalena L, Martino E \& Bogazzi F. The onset time of amiodarone-induced thyrotoxicosis (AIT) depends on AIT type. European Journal of Endocrinology/European Federation of Endocrine Societies 2014171 363-368. (doi:10.1530/eje-14-0267)
6 Yiu KH, Jim MH, Siu CW, Lee CH, Yuen M, Mok M, Shea YF, Fan $\mathrm{K}$, Tse HF \& Chow WH. Amiodarone-induced thyrotoxicosis is a predictor of adverse cardiovascular outcome. Journal of Clinical Endocrinology and Metabolism 200994 109-114. (doi:10.1210/jc.20081907)

7 Bogazzi F, Tomisti L, Bartalena L, Aghini-Lombardi F \& Martino E. Amiodarone and the thyroid: a 2012 update. Journal of Endocrinological Investigation 201235 340-348. (doi:10.3275/8298)

8 Tanda ML, Piantanida E, Lai A, Liparulo L, Sassi L, Bogazzi F, Wiersinga WM, Braverman LE, Martino E \& Bartalena L. Diagnosis and management of amiodarone-induced thyrotoxicosis: similarities and differences between North American and European thyroidologists. Clinical Endocrinology 200869 812-818. (doi:10.1111/ j.1365-2265.2008.03268.x)

9 Ahmed S, Ranchor AV, Crijns HJ, Van Veldhuisen DJ, Van Gelder IC \& CONVERT investigators. Effect of continuous versus episodic amiodarone treatment on quality of life in persistent atrial fibrillation. Europace 201012 785-791. (doi:10.1093/europace) euq049)

10 Ahmed S, Van Gelder IC, Wiesfeld AC, Van Veldhuisen DJ \& Links TP. Determinants and outcome of amiodarone-associated thyroid dysfunction. Clinical Endocrinology 201175 388-394. (doi:10.1111/ j.1365-2265.2011.04087.x)

11 Trip MD, Wiersinga W \& Plomp TA. Incidence, predictability, and pathogenesis of amiodarone-induced thyrotoxicosis and hypothyroidism. American Journal of Medicine 199191 507-511. (doi:10.1016/0002-9343(91)90187-3)

12 Eskes SA, Endert E, Fliers E, Geskus RB, Dullaart RP, Links TP \& Wiersinga WM. Treatment of amiodarone-induced thyrotoxicosis type 2: a randomized clinical trial. Journal of Clinical Endocrinology and Metabolism 201297 499-506. (doi:10.1210/jc.2011-2390)

13 Bahn Chair RS, Burch HB, Cooper DS, Garber JR, Greenlee MC, Klein I, Laurberg P, McDougall IR, Montori VM, Rivkees SA et al. Hyperthyroidism and other causes of thyrotoxicosis: management guidelines of the American Thyroid Association and American Association of Clinical Endocrinologists. Thyroid 201121 593-646. (doi:10.1089/thy.2010.0417)

14 Ross DS, Burch HB, Cooper DS, Greenlee MC, Laurberg P, Maia AL, Rivkees SA, Samuels M, Sosa JA, Stan MN et al. 2016 American Thyroid Association Guidelines for Diagnosis and Management of Hyperthyroidism and Other Causes of Thyrotoxicosis. Thyroid: Official Journal of the American Thyroid Association 201626 1343-1421. (doi:10.1089/thy.2016.0229)

15 Hermus A, Dekkers O, Berghout A, Muller A, Wiersinga W, Peeters R \& Ploeg van der M. Richtlijn Schildklierfunctiestoornissen Revisie 2012 2012 101-106.

16 Eskes SA \& Wiersinga WM. Amiodarone and thyroid. Best practice \& Research. Clinical Endocrinology \& Metabolism 200923 735-751. (doi:10.1016/j.beem.2009.07.001)

17 Sato K, Shiga T, Matsuda N, Onoda N, Takano K, Hagiwara N \& Kasanuki H. Mild and short recurrence of type II amiodarone-induced thyrotoxicosis in three patients receiving amiodarone continuously for more than 10 years. Endocrine Journal 200653 531-538. (doi:10.1507/endocrj.k06-027)

18 Bogazzi F, Bartalena L, Tomisti L, Rossi G, Brogioni S \& Martino E. Continuation of amiodarone delays restoration of euthyroidism in patients with type 2 amiodarone-induced thyrotoxicosis treated with prednisone: a pilot study. Journal of Clinical Endocrinology and Metabolism 201196 3374-3380. (doi:10.1210/jc.2011-1678)

19 Kim HL, Seo JB, Chung WY, Kim SH, Kim MA \& Zo JH. The incidence and predictors of overall adverse effects caused by low dose amiodarone in real-world clinical practice. Korean Journal of Internal Medicine 201429 588-596. (doi:10.3904/kjim.2014.29.5.588)

20 Tomisti L, Materazzi G, Bartalena L, Rossi G, Marchello A, Moretti M, De Napoli L, Mariotti R, Miccoli P, Martino E et al. Total thyroidectomy in patients with amiodarone-induced thyrotoxicosis and severe left 
ventricular systolic dysfunction. Journal of Clinical Endocrinology and Metabolism 201297 3515-3521. (doi:10.1210/jc.2012-1797)

21 Conen D, Melly L, Kaufmann C, Bilz S, Ammann P, Schaer B, Sticherling C, Muller B \& Osswald S. Amiodarone-induced thyrotoxicosis: clinical course and predictors of outcome. Journal of the American College of Cardiology 200749 2350-2355. (doi:10.1016/j. jacc.2007.02.054)

22 Barvalia U, Amlani B \& Pathak R. Amiodarone-induced thyrotoxic thyroiditis: a diagnostic and therapeutic challenge. Case Reports in Medicine 20142014 231651. (doi:10.1155/2014/231651)
23 Hashimoto K, Ota M, Irie T, Takata D, Nakajima T, Kaneko Y, Tanaka Y, Matsumoto S, Nakajima Y, Kurabayashi M et al. A case of type 2 amiodarone-induced thyrotoxicosis that underwent total thyroidectomy under high-dose steroid administration. Case Reports in Endocrinology 20152015416145. (doi:10.1155/2015/416145)

24 Hofmann A, Nawara C, Ofluoglu S, Holzmannhofer J, Strohmer B $\&$ Pirich C. Incidence and predictability of amiodarone-induced thyrotoxicosis and hypothyroidism. Wiener Klinische Wochenschrift 2008120 493-498. (doi:10.1007/s00508-008-1017-2)

Received 9 January 2017

Revised version received 10 April 2017

Accepted 19 April 2017 